\title{
Clinical Pearl:
}

\section{Case Report: Congenital Syphilis and Cytomegalovirus Infection in a Mother with Substance Use Disorder: Case-Based Review}

Yessenia Castro-Caballero, MD, Harshil Matta , MD, Joseph Hageman, MD

\begin{abstract}
:
The incidence of congenital syphilis in the United States is rising. No prenatal care or lack of early detection may be a cause for the rise in this serious yet preventable and treatable disease. Here we review a case, that through physical findings, sparked an index of suspicion to promptly test and treat for congenital syphilis as well

\section{"Congenital syphilis is an infectious disease caused by the spirochete it in utero prior to birth. This mode is the most common form of transmission. Early diagnosis and detection of congenital syphilis is key to preventing further sequela."} treponema palladium. The infant acquires
\end{abstract}

as congenital CMV.

\section{Introduction:}

Congenital syphilis is an infectious disease caused by the spirochete treponema palladium. The infant acquires it in utero prior to birth. This mode is the most common form of transmission. Early diagnosis and detection of congenital syphilis is key to preventing further sequela. Making the diagnosis can be difficult as the presentation can be vague and nonspecific at birth. Even symptomatic infants can be challenging to diagnose due to the subtle nature of the symptoms or atypical presentations. Recognizing congenital syphilis and becoming familiar with its presentation is essential to early diagnosis and initiation of treatment. Congenital syphilis is on the rise in the United States, and recent data suggest a surge in incidence. Here we report a case of congenital syphilis in a newborn infant.

\section{Case Presentation:}

A newborn female infant was born to a woman of advanced maternal age, at term gestation, 35-year-old G5P3023 at 39 2/7 weeks gestational age via cesarean -section. Mother had very poor prenatal care with few visits prior to arriving at labor and delivery. The mother reported multiple drug use with cocaine, heroin, and alcohol throughout the pregnancy. The mother admits to snorting heroine 2 hours prior to arrival at labor and delivery. She was taking prenatal vitamins during pregnancy and had no other significant past medical history. Prenatal labs include blood type $O$ positive, antibody negative, GBS unknown, RPR unknown pending, rubella unknown pending, HIV negative, COVID-19 negative at delivery.

A female infant was born via cesarean section with meconium- stained fluid at rupture. The infant was brought to a warmer, cleaned, stimulated, and suctioned. The infant was noted to be coughing up thick meconium. Initial lung examination displayed course lung sounds. The infant was deep suctioned, and CPAP 5L and $\mathrm{FiO}_{2} 21 \%$ were initiated as subcostal retractions were present. Oxygen saturation at this point was $77 \%$, FIO2 was increased to $30 \%$, and the infant was transferred to the nursery where she was weaned to 2 LPM nasal cannula (NC). Chest x-rays revealed findings consistent with retained fetal lung fluid or transient tachypnea of the newborn (TTN). Apgars were 8 and 9 at one and five minutes of age.

On examination, infant weight was 3560 grams, head circumference $34.5 \mathrm{~cm}$, length $48.5 \mathrm{~cm}$. The infant was vigorous but in mild respiratory distress. A cardiac exam revealed a systolic 3/6 murmur present at LUSB and LLSB. The tone was normal. However, the infant displayed intermittent tremors of the upper and lower extremities. Skin exam revealed firm purple nodules on the right forehead, left eyelid, chin, and left foot. Petechiae were present on the chest and lower legs. Infant oxygen saturations were stable on 2 LPM NC, 30\% oxygen. Initial complete blood count (CBC) had a platelet count of 25,000; repeat platelet count was 21,000. At this time infant was transferred for TORCH workup, platelet transfusion, septicemia diagnostic workup. The infant was started on intravenous Ampicillin and Gentamicin.

Assessment: The diagnosis was established with the rest of the TORCH diagnostic workup.

\section{Clinical Course:}

The patient received one platelet transfusion with the subsequent platelet count recovery to 100,000 on postnatal life 13 . The patient was weaned off oxygen in two days. The patient developed neonatal abstinence syndrome managed with non-pharmacologic care along with three doses of PRN morphine. Maternal laboratory studies included $r$ FTA antibody reactive, RPR Reflex QT +TPPA reactive, and RPR titer $1: 4$. Infant treponemal antibody resulted positive with RPR 1:2., the maternal titer and mother were not treated given a serum quantitative nontreponemal serologic titer equal to or less than fourfold; however, the infant was diagnosed as possible congenital syphilis, and IV penicillin was started. CSF studies were obtained. CSF VDRL was negative. CSF meningitis panel for bacteria, viruses, and yeast was negative. CSF count: total RBCs 380, WBCs 12 with Seg \% 22, L \% 46. Infant quantitative CMV of urine $>6.30$, which was positive for Cytomegalovirus infection. Treatment with valganciclovir was initiated and continued for six months. Blood, urine, and CSF culture had no growth. Long bone X-rays showed no periostitis, destructive lesions, or other evidence of syphilis. Head ultrasound showed no brain parenchymal abnormality or calcifications and no evidence of germinal matrix hemorrhage. An ophthalmologic exam showed no signs of CMV retinitis. The patient finished a 10-day course of IV penicillin. The patient did well throughout the hospital stay and was discharged home in healthy condition on day of life 25. Follow-up visits included RPR monitoring. At three months, RPR was 1:4, at four months follow-up RPR reactive at 1:1, and 6-month follow-up non-reactive. Physical examination, feeding, and growth review displayed a thriving infant progressing toward full recovery.

\section{Discussion:}



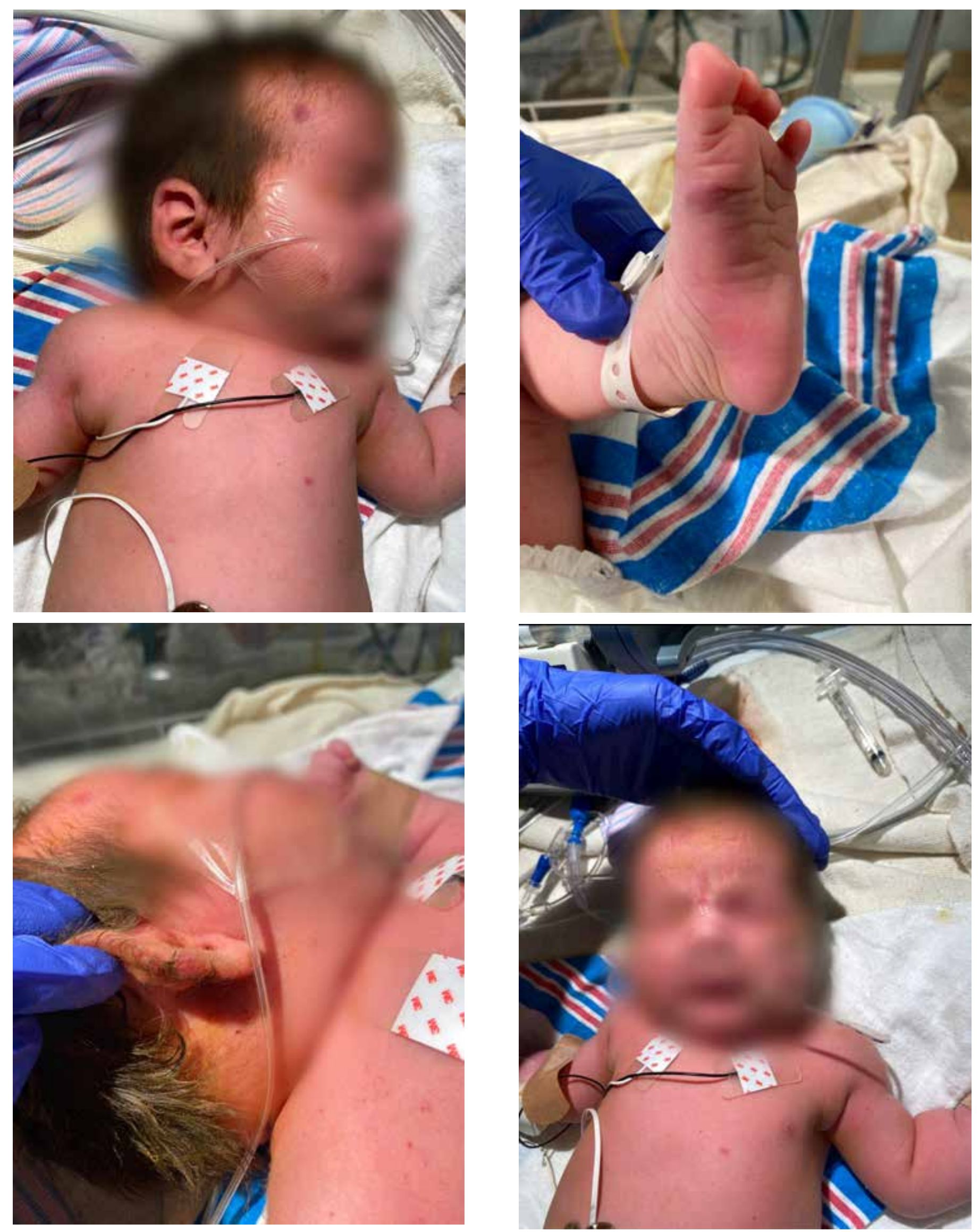
Congenital syphilis is on the rise in the United States, and recent data suggest a surge in incidence. CDC reported a four-fold increase in cases of congenital syphilis from 2013 to 2018 in the United States, going from 362 to 1306 cases. In 2018 the rate of congenital syphilis was 33.1 per 100,000 births, a tremendous surge from 2014 where the case rate was 11.6 per 100,000 births. Congenital syphilis can result in catastrophic sequela of spontaneous abortion, intrauterine growth restriction (IUGR), stillbirth, prematurity, hydrops fetalis, and death in newborn infants.

Congenital syphilis is categorized broken into early and late. In early congenital syphilis, the most common findings are hepatomegaly, jaundice, snuffles, rash, skeletal abnormalities, fever, sepsis. Approximately 60-90 percent of infants are asymptomatic at birth. Symptomatic infants may further present with anemia, thrombocytopenia, and respiratory complications. Manifestations of late congenital syphilis include saddle nose deformity, frontal bossing, Hutchinson's triad, anterior bowing of shins.

Testing for congenital syphilis involves RPR testing and treponemal tests. The most common manifestation of early congenital syphilis is the rash. The index of suspicion should be raised when the rash is combined with poor prenatal care, thrombocytopenia, respiratory complications, and failure to thrive. In our patient, the initial presentation included rash, respiratory compromise, and thrombocytopenia combined with high-risk maternal behavior and poor prenatal care. RPR titers and treponemal testing pointed toward congenital syphilis in our patient with prompt treatment of penicillin initiated.

\section{"Our developed nation is not immune} to congenital syphilis, and it is more important now to recognize, diagnose and treat congenital syphilis as early as possible to prevent catastrophic sequelae. Physicians should be aware of the sometimes vague presentation of early congenital syphilis and display a high index of suspicion when evaluating infants in the newborn period."

\section{Conclusion:}

The cases of congenital syphilis in the United States are rising, with a four-fold increase from 2013 to 2018. Currently, cases sit at 33.1 per 100,000 births. Our developed nation is not immune to congenital syphilis, and it is more important now to recognize, diagnose and treat congenital syphilis as early as possible to prevent catastrophic sequelae. Physicians should be aware of the sometimes vague presentation of early congenital syphilis and display a high index of suspicion when evaluating infants in the newborn period.

\section{References:}

1. Kwak J, Lamprecht C. A review of the guidelines for the evaluation and treatment of congenital syphilis. Pediatric Annals 2015; 44(5): e108-e114.

2. Davis NL, King CC, Kourtis AP. Cytomegalovirus infection in pregnancy. Birth Defects Research 2017; 109: 336346.

3. Penner, J et al. Stop, thing SCORTCH: rethinking the traditional "TORC

Disclosures: The authors have no disclosures

NT
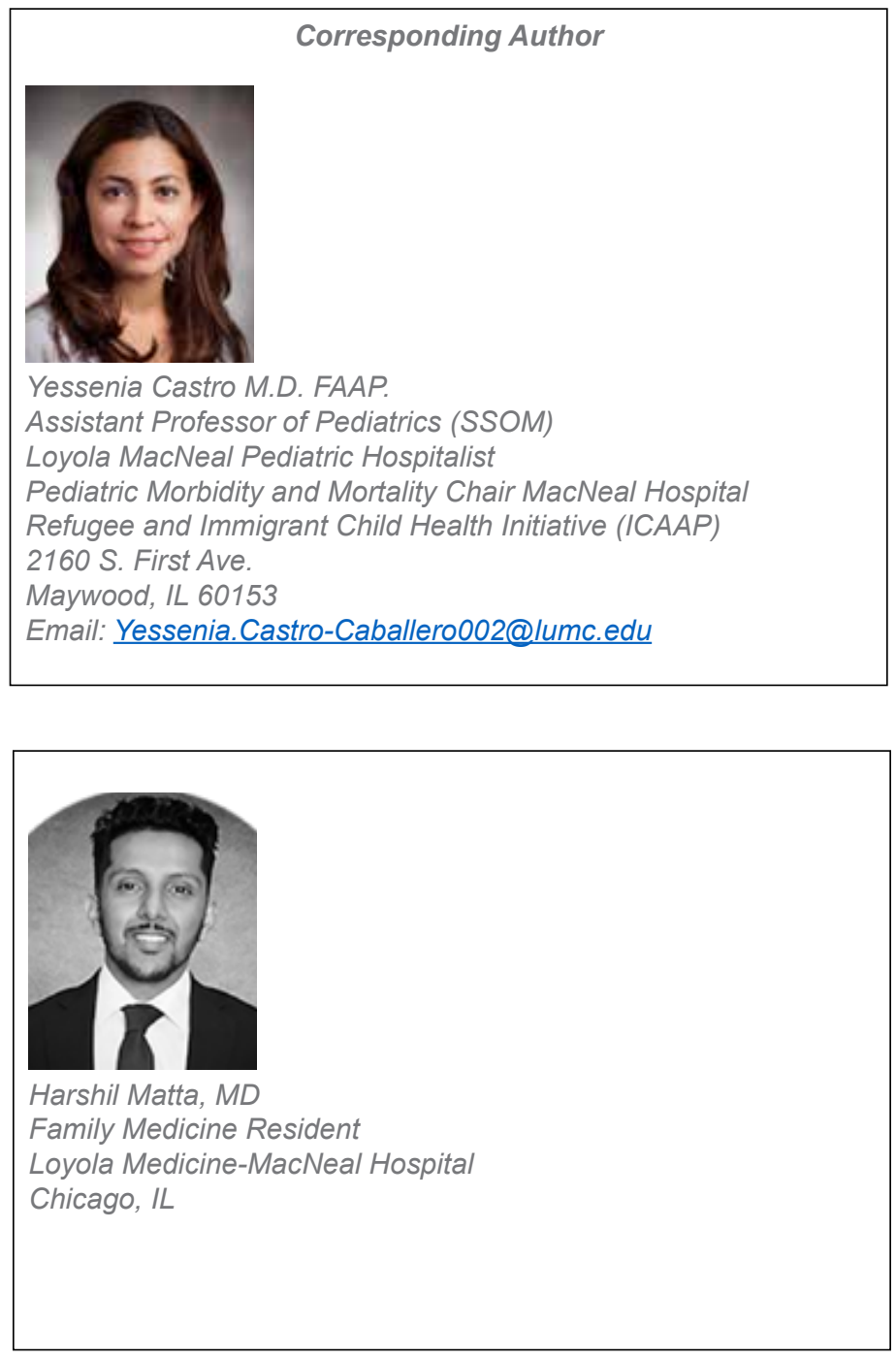

NEONATOLOGY TODAY is interested in publishing manuscripts from Neonatologists, Fellows, NNPs and those involved in caring for neonates on case studies, research results, hospital news, meeting announcements, and other pertinent topics.

Please submit your manuscript to: LomaLindaPublishingCompany@gmail.com 


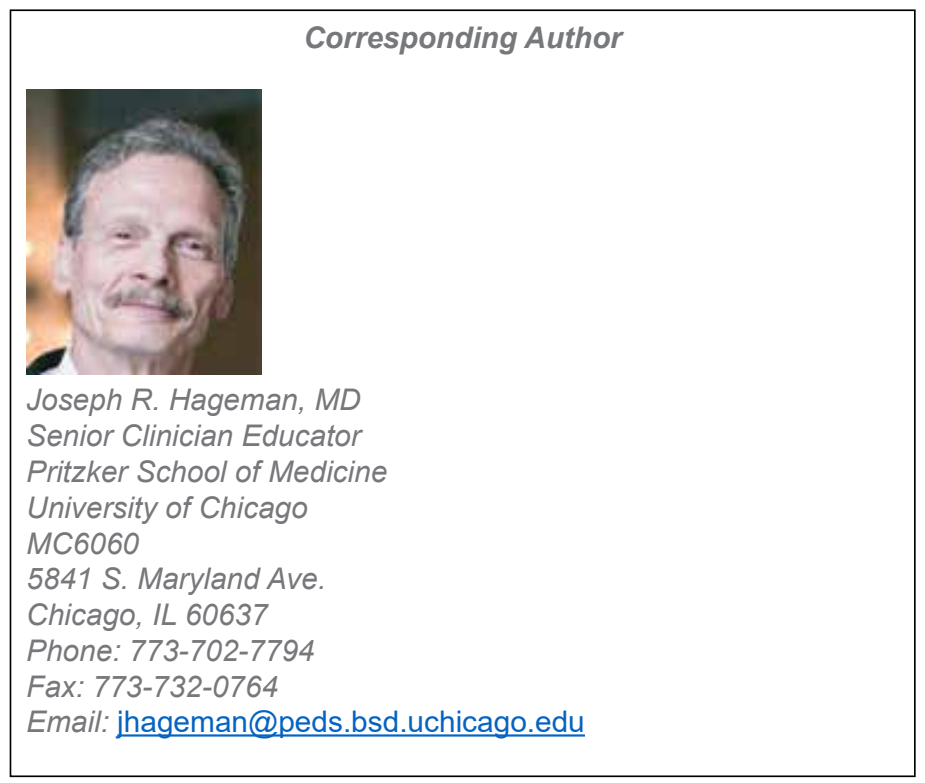

$\underline{\text { Clinical Pearls are published monthly. }}$

Submission guidelines for "Clinical Pearls":

1250 word limit not including references or title page.

May begin with a brief case summary or example.

Summarize the pearl for emphasis.

No more than 7 references.

Please send your submissions to:

jhageman@peds.bsd.uchicago.edu

\section{Readers can also follow NEONATOLOGY \\ via our Twitter Feed @NEO}

Which Infants are More Vulnerable to Respiratory Syncytial Virus?

\begin{abstract}
RSV is a respiratory virus with cold-like symptoms that causes 90,000 hospitalizations and 4,500 deaths per year in children

5 and younger. It's 10 times more deadly than the flu.

For premature babies with fragile immune systems and

underdeveloped lungs, RSV proves especially dangerous.
\end{abstract}

But risk factors associated with RSV don't touch all infants equally.*

\begin{tabular}{|c|c|c|}
\hline Caucasian Babies & Risk Factor & $\begin{array}{c}\text { African American } \\
\text { Babies }\end{array}$ \\
\hline $11.6 \%$ & Prematurity & $18.3 \%$ \\
\hline $58.1 \%$ & Breastfeeding & $50.2 \%$ \\
\hline $7.3 \%$ & $\begin{array}{l}\text { Low Birth } \\
\text { Weight }\end{array}$ & $11.8 \%$ \\
\hline $60.1 \%$ & Siblings & $71.6 \%$ \\
\hline $1 \%$ & $\begin{array}{l}\text { Crowded } \\
\text { Living } \\
\text { Conditions }\end{array}$ & $3 \%$ \\
\hline
\end{tabular}

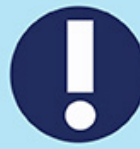

AFRICAN AMERICAN BABIES bear the brunt of RSV. Yet the American Academy of Pediatrics' restrictive new guidlines limit their access to RSV preventative treatment, increasing these babies' risk.

$$
\mathrm{A} f \mathrm{PA}
$$

\section{FREE for our NICU COMMUNITY}

- Helping Children and Families Cope

- Bonding with Your Baby

- Caregivers Need Care Too
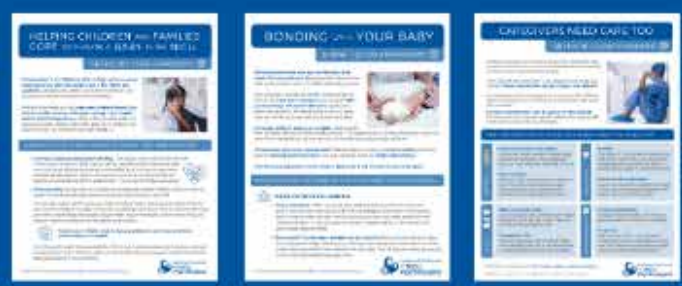